\title{
Normal State of a Polarized Fermi Gas at Unitarity
}

\author{
C. Lobo, A. Recati, S. Giorgini, and S. Stringari \\ Dipartimento di Fisica, Università di Trento and CNR-INFM BEC Center, I-38050 Povo, Trento, Italy
}

(Received 27 July 2006; published 13 November 2006)

\begin{abstract}
We study the Fermi gas at unitarity and at $T=0$ by assuming that, at high polarizations, it is a normal Fermi liquid composed of weakly interacting quasiparticles associated with the minority spin atoms. With a quantum Monte Carlo approach we calculate their effective mass and binding energy, as well as the full equation of state of the normal phase as a function of the concentration $x=n_{\downarrow} / n_{\uparrow}$ of minority atoms. We predict a first order phase transition from normal to superfluid at $x_{c}=0.44$ corresponding, in the presence of harmonic trapping, to a critical polarization $P_{c}=\left(N_{\uparrow}-N_{\downarrow}\right) /\left(N_{\uparrow}+N_{\downarrow}\right)=77 \%$. We calculate the radii and the density profiles in the trap and predict that the frequency of the spin dipole mode will be increased by a factor of 1.23 due to interactions.
\end{abstract}

DOI: 10.1103/PhysRevLett.97.200403

PACS numbers: 05.30.Fk, 03.75.Hh, 03.75.Ss

Recent experiments on degenerate gases of ${ }^{6} \mathrm{Li}$ with a mixture of two hyperfine species have explored the physics of Fermi gases [1,2] and have led to a number of theoretical analyses [3-7]. One of the major experimental observations has been the occurrence of phase separation if the mixture contains more atoms of one species than of the other, i.e., if the gas is polarized. Some of the experiments suggest that in the unitary limit of strong interactions there are three phases: an unpolarized superfluid phase, a mixed phase which exhibits a partial polarization, and a fully polarized gas. We now have a good understanding of the superfluid phase which has been the subject of numerous theoretical and experimental studies while the fully polarized phase is an ideal Fermi gas since atoms in the same spin state do not interact with each other. However, for intermediate polarizations, when both species are present, the nature of the mixed phase is not understood.

Here we study the mixed phase in the unitary limit by adopting an approach inspired by the theory of dilute solutions of ${ }^{3} \mathrm{He}$ in ${ }^{4} \mathrm{He}$ [8]. We will assume that the majority species $(\uparrow)$ forms a background experienced by the minority species $(\downarrow)$ and that the latter behaves as a gas of weakly interacting fermionic quasiparticles even though the $\uparrow-\downarrow$ atomic interaction is very strong, being characterized by an infinite scattering length. In other words, we will assume that the system is a normal Fermi liquid, which will allow us to characterize the energy of the gas in terms of a few parameters and, by calculating these with a quantum Monte Carlo approach, to make various predictions of experimental relevance.

We begin by writing the expression for the energy $E$ of a homogeneous system in the limit of very dilute mixtures and at zero temperature. The concentration of $\downarrow$ atoms is given by the ratio of the densities $x=n_{\downarrow} / n_{\uparrow}$, and we will take it to be small. If only $\uparrow$ atoms are present, then the energy is that of an ideal Fermi gas $E(x=0)=3 / 5 E_{F \uparrow} N_{\uparrow}$, where $N_{\uparrow}$ is the total number of $\uparrow$ atoms and $E_{F \uparrow}=$ $\hbar^{2} / 2 m\left(6 \pi^{2} n_{\uparrow}\right)^{2 / 3}$ is the ideal gas Fermi energy. When we add a $\downarrow$ atom with a momentum $\mathbf{p}\left(|\mathbf{p}| \ll p_{F \uparrow}\right)$, we shall assume that the change in $E$ is given by

$$
\delta E=\frac{p^{2}}{2 m^{*}}-\frac{3}{5} E_{F \uparrow} A .
$$

In other words, the $\downarrow$ atom in the $\uparrow$ gas behaves as a quasiparticle with a quadratic spectrum and an effective mass $m^{*}$. In addition, there is a "binding" energy $-3 / 5 E_{F \uparrow} A$ of the $\downarrow$ atom to the Fermi gas of $\uparrow$ atoms. This binding energy must be proportional to $E_{F \uparrow}$ since there is no other energy scale in the unitary limit, and we have used the factor $3 / 5$ for later convenience. We shall further assume that this quasiparticle is a fermion [9].

When we add more $\downarrow$ atoms, creating a small finite density $n_{\downarrow}$, they will form a degenerate gas of quasiparticles at zero temperature occupying all the states with momentum up to the Fermi momentum $p_{F \downarrow}=\hbar\left(6 \pi^{2} n_{\downarrow}\right)^{1 / 3}$. The energy of the system can then be written in a useful form in terms of the concentration $x$ as

$$
\frac{E(x)}{N_{\uparrow}}=\frac{3}{5} E_{F \uparrow}\left(1-A x+\frac{m}{m^{*}} x^{5 / 3}\right) .
$$

Equation (2) is valid for small values of the concentration $x$, i.e., when interactions between $\downarrow$ quasiparticles as well as further renormalization effects of the parameters can be neglected.

In the following, we will calculate $A$ and $m^{*}$ using a fixed-node diffusion Monte Carlo (FN-DMC) approach, already employed in earlier studies [10,11]. We use the same attractive square-well potential to model the interactions between $\downarrow$ and $\uparrow$ atoms: $V(r)=-V_{0}$ for $r<R_{0}$ and $V(r)=0$ otherwise. The short range $R_{0}$ is chosen as $2 n_{\uparrow} R_{0}^{3}=10^{-6}$. The depth $V_{0}$ is fixed by the unitarity condition $|a|=\infty$ for the $s$-wave scattering length $a$ and corresponds to the threshold for the first two-body bound state in the well: $V_{0}=\pi^{2} \hbar^{2} /\left(4 m R_{0}^{2}\right)$. For a single $\downarrow$ atom in a homogeneous Fermi sea of $\uparrow$ atoms the trial wave function $\psi_{T}$, which determines the nodal surface used as an 
ansatz in the FN-DMC calculation, is chosen to be of the form [12]

$$
\psi_{T}^{\mathbf{q}}\left(\mathbf{r}_{\downarrow}, \mathbf{r}_{1 \uparrow}, \ldots, \mathbf{r}_{N_{\uparrow}}\right)=\exp \left(i \mathbf{q} \cdot r_{\downarrow}\right) \prod_{i=1}^{N_{\uparrow}} f\left(r_{i \downarrow}\right) D_{\uparrow}\left(N_{\uparrow}\right),
$$

where $r_{\downarrow}$ denotes the position of the $\downarrow$ atom and $r_{i \downarrow}=$ $\left|\mathbf{r}_{\downarrow}-\mathbf{r}_{i \uparrow}\right|$. In this equation the plane wave $\exp \left(i \mathbf{q} \cdot r_{\downarrow}\right)$ corresponds to the impurity traveling through the medium with momentum $\hbar \mathbf{q}=\left(n_{x}, n_{y}, n_{z}\right) 2 \pi \hbar / L$, where $L$ is the length of the cubic box and the $n_{i}$ are integers describing the momentum in each coordinate. Furthermore, $D_{\uparrow}\left(N_{\uparrow}\right)$ is the Slater determinant of plane waves describing the Fermi sea of the $N_{\uparrow}$ atoms and the Jastrow term $f(r)$ accounts for correlations between the impurity and the Fermi sea. The correlation function $f(r)$ is chosen as in Ref. [10]. We consider a system of $N_{\uparrow}=33$ atoms and periodic boundary conditions. From the energy $E$ of the system with $N_{\uparrow}+1$ atoms, we subtract the exact energy $E_{0}\left(N_{\uparrow}\right)$ of the Fermi sea. The result is shown in Fig. 1 as a function of $\left(q / k_{F \dagger}\right)^{2}$. From a linear best fit we obtain the following values: $A=$ $0.97(2)$ and $m^{*} / m=1.04(3)$, which is consistent with the general inequality $m^{*} / m \geq 1$. We have checked that finitesize corrections associated with the number $N_{\uparrow}$ of atoms in the Fermi sea are below the reported statistical error. It is worth noticing that the binding energy of a single $\downarrow$ atom almost coincides with the average energy of an atom in the Fermi sea. A similar coincidence was found for the superfluid gap $\Delta$ at unitarity [13].

A relevant question is to understand whether the equation of state (2) is adequate to describe regimes of large values of $x$ where interaction between quasiparticles and other effects might become important. To answer this question we have carried out a FN-DMC calculation of the equation of state at finite concentrations $x=N_{\downarrow} / N_{\uparrow}$ using the trial wave function

$$
\psi_{T}\left(\mathbf{r}_{1^{\prime}}, \ldots, \mathbf{r}_{N_{\downarrow}}, \mathbf{r}_{1}, \ldots, \mathbf{r}_{N_{\uparrow}}\right)=\prod_{i, i^{\prime}} f\left(r_{i i^{\prime}}\right) D_{\downarrow}\left(N_{\downarrow}\right) D_{\uparrow}\left(N_{\uparrow}\right),
$$

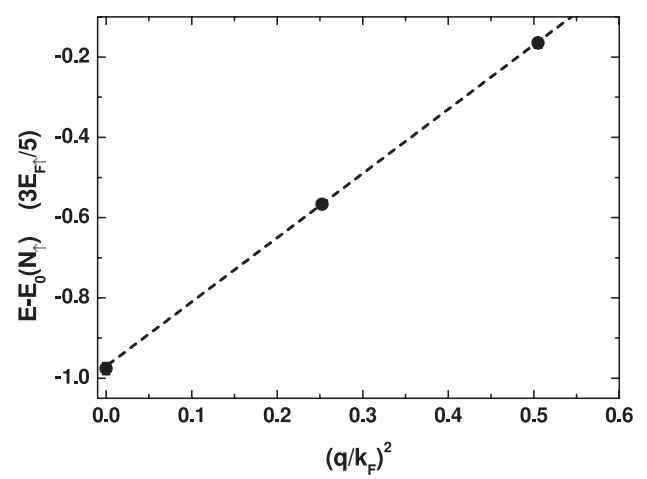

FIG. 1. Excitation spectrum of a $\downarrow$ atom in a Fermi sea of $\uparrow$ atoms. The dashed line is a linear best fit to the FN-DMC results from which we extract the values $A$ and $m^{*}$. where $i$ and $i^{\prime}$ label, respectively, $\uparrow$ and $\downarrow$ atoms. The nodal surface of the wave function $\psi_{T}$ is determined by the product of Slater determinants $D_{\uparrow}\left(N_{\uparrow}\right) D_{\downarrow}\left(N_{\downarrow}\right)$ and coincides with the nodal surface of a two-component ideal Fermi gas. As a consequence, the wave function in Eq. (4) is incompatible with off-diagonal long-range order (ODLRO) and describes a normal Fermi gas. In contrast, the BCStype wave function used in Refs. [10,11] is compatible with ODLRO and describes a superfluid state. A direct comparison between the ground state energy of the normal and superfluid states can be carried out for equal numbers of $\uparrow$ and $\downarrow$ atoms, $N_{\downarrow}=N_{\uparrow}$, with the result $E_{\mathrm{SF}} /\left(3 / 5 E_{F \uparrow} N_{\uparrow}\right)=0.84(2)$ and $E_{\mathrm{N}} /\left(3 / 5 E_{F \uparrow} N_{\uparrow}\right)=1.12(2)$ (see also [13]) showing the instability of the normal state for $x=1$ (see Fig. 2).

The results for the equation of state of the normal Fermi gas are shown in Fig. 2. To reduce finite-size effects we have considered closed-shell configurations $N_{\downarrow}=$ 7, 19, 27, 33 with $N_{\uparrow}=27,33$. In Fig. 2 we also show the prediction of Eq. (2) based on noninteracting quasiparticles (dashed line). For small values of $x$ we find very good agreement, but for larger concentrations effects of interactions between quasiparticles start to be important and deviations from Eq. (2) become visible. The solid line is obtained from a polynomial best fit to the FN-DMC results.

From the equation of state of the mixed phase we can determine the transition between the fully polarized and the mixed phases as well as the transition between the mixed and the unpolarized superfluid phases [14]. The equilibrium condition is obtained by imposing that the chemical potential and the pressure be the same in the two phases. It is useful to express the results in terms of the chemical potential $\mu=\left(\mu_{\uparrow}+\mu_{\downarrow}\right) / 2$ and the effective magnetic field $h=\left(\mu_{\uparrow}-\mu_{\downarrow}\right) / 2$. Here $\mu_{\uparrow \downarrow}=\partial E / \partial N_{\uparrow \downarrow}$ is the chemical potential of each spin species. The transition

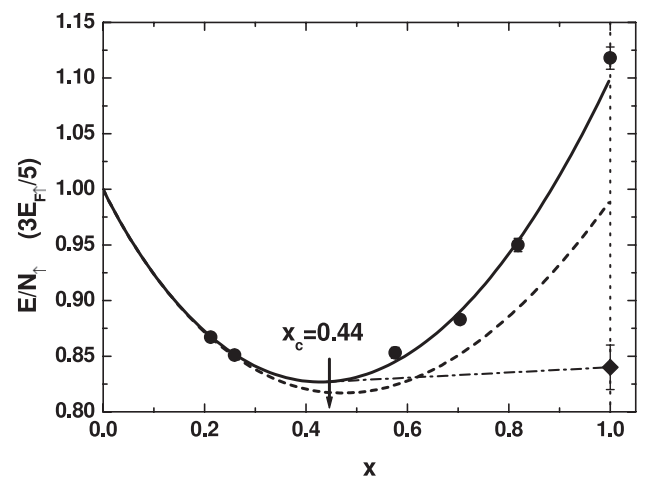

FIG. 2. Equation of state of a normal Fermi gas as a function of the concentration $x$ (circles). The solid line is a polynomial best fit to the FN-DMC results. The dashed line corresponds to Eq. (2). The dot-dashed line is the coexistence line between the normal and the unpolarized superfluid states, and the arrow indicates the critical concentration $x_{c}$ above which the system phase separates. For $x=1$, the energy of both the normal and the superfluid (diamond) states are shown. 
between the fully polarized and the mixed phase is second order and takes place at $x=0$ where we find $\mu_{\downarrow} / \mu_{\uparrow}=$ $-3 / 5 A$, corresponding to $h / \mu=3.78$. The transition between the mixed and the unpolarized superfluid phases is instead first order and the coexistence curve is shown in Fig. 2 (see also [7]). We obtain the critical value $x_{c}=0.44$ at the transition. For smaller values of $x$ the system remains in the normal state, while above the critical concentration $x_{c}$ the system will begin nucleating the superfluid and phase separate into those two states. The phase transition is characterized by the value $\mu_{\downarrow} / \mu_{\uparrow}=0.017$ corresponding to $h / \mu=0.96$ [15]. Note that at the critical value $x_{c}=$ 0.44 the difference between the best fit and the prediction of Eq. (2) is quite small so that this latter energy functional describes well the whole normal phase. This supports the idea that the normal state is a gas of weakly interacting quasiparticles. Equation (2) would actually predict the value $x_{c}=0.50$ for the critical concentration, which corresponds to $h / \mu=0.93$. In this respect, we notice that the Fermi pressure term in $x^{5 / 3}$ plays a crucial role.

It is useful to compare the above results with BCS theory which also predicts 3 phases at unitarity [4,5]: a superfluid with energy $E_{\mathrm{BCS}} /\left(3 / 5 E_{F \uparrow} N_{\uparrow}\right)=1.18$, a mixed state which is a noninteracting partially polarized gas, and a fully polarized gas. The mixed state energy is simply the kinetic energy and is an increasing function of $x$. In this case $x_{c}=$ 0.04 , corresponding to $\mu_{\uparrow} / \mu_{\downarrow}=0.1$, thereby leading to a much reduced normal region with respect to the predictions of our FN-DMC calculation.

Now we turn to the trapped case. While we will discuss the situation only at zero temperature, we should note that temperature can have an important effect on the density profile of the $\downarrow$ atoms. In experiments we usually have the condition $k_{B} T \ll E_{F \uparrow}$. But since $n_{\downarrow} \ll n_{\uparrow}$, we might be in a situation where $k_{B} T \geqslant E_{F \downarrow}$ and would therefore need to use the appropriate thermal distribution. With this caveat we turn to the inhomogeneous situation where we shall use the local density approximation (LDA) [16]. In a harmonic trap with potential $V(\mathbf{r})=\sum_{i} m \omega_{i}^{2} r_{i}^{2} / 2$, the local chemical potentials become $\mu_{\uparrow \downarrow}(\mathbf{r})=\mu_{\uparrow \downarrow}^{0}-V(\mathbf{r})$. For small concentrations (in particular, $x \ll x_{c}$ ), where only the normal state is present and where we can neglect the change in $\mu_{\uparrow}$ due to the attraction of the $\downarrow$ atoms, $n_{\uparrow}$ is the ThomasFermi density of an ideal gas whereas $n_{\downarrow}$ is a ThomasFermi profile with a modified harmonic potential $V \rightarrow V \times$ $(1+3 / 5 A)$. The potential seen by the $\downarrow$ atoms is more confining due to the attraction to the $\uparrow$ atoms. This also has consequences for the collective modes of the system: it leads us to predict that the spin dipole mode - the mode where the $\downarrow$ atoms oscillate as a whole in the midst of the $\uparrow$ atom cloud - will have a frequency given by

$$
\omega_{i}^{\mathrm{dip}}=\omega_{i} \sqrt{(1+3 / 5 A) \frac{m}{m^{*}}} \simeq 1.23 \omega_{i}
$$

So, a direct measurement of the oscillation frequency of the $\downarrow$ atoms in a dilute mixture with $x \ll 1$ would provide a useful test of this Fermi liquid theory and, in particular, of the numerical estimate of the parameters $A$ and $m^{*}$ [17]. For larger concentrations the system will exhibit a central superfluid core with chemical potential $\mu_{\mathrm{SF}}=\left(\mu_{\uparrow}+\right.$ $\left.\mu_{\downarrow}\right) / 2$, whose radius in a spherical trap is given by

$$
R_{\mathrm{SF}}^{2}=R_{\uparrow}^{2} \frac{\mu_{\downarrow}^{0} / \mu_{\uparrow}^{0}-\mu_{\downarrow} / \mu_{\uparrow}}{1+\mu_{\downarrow} / \mu_{\uparrow}},
$$

where $R_{\uparrow}$ is the radius of the $\uparrow$ component and $\mu_{\downarrow} / \mu_{\uparrow}$ is calculated at the transition point. In Fig. 3, we plot the radius $R_{\mathrm{SF}}$ of the superfluid component together with the radii of the minority and majority components, $R_{\uparrow}$ and $R_{\downarrow}$, respectively, in units of $R_{\uparrow}^{0}=a_{\mathrm{ho}}\left(48 N_{\uparrow}\right)^{1 / 6}$ as a function of the polarization $P=\left(N_{\uparrow}-N_{\downarrow}\right) /\left(N_{\uparrow}+N_{\downarrow}\right)$ of the sample. We predict that the superfluid phase disappears at $P_{c}=$ 0.77 in good agreement with the experimental findings of [2]. Notice that as $P \rightarrow 1, R_{\downarrow} \sim R_{\uparrow}^{0}\left[(1+3 / 5 A) m^{*} / m^{-1 / 4} \times\right.$ $[(1-P) /(1+P)]^{1 / 6} \rightarrow 0$, while $R_{\uparrow}$ approaches the noninteracting value. In the opposite $P \rightarrow 0$ limit, the radii converge to the known value $\left(\mu / E_{F \uparrow}\right)^{1 / 4} \simeq 0$.80. It is worth noticing that the BCS approach would predict the value $P_{c}>0.99$ at unitarity [4,5], pointing out the dramatic role played by the binding energy in the mixed state.

At the transition between the superfluid and the mixed normal phase the densities of the two spin species exhibit a discontinuity, revealing the first order nature of the transition. The densities $n_{\uparrow}$ and $n_{\downarrow}$ jump from the superfluid value $n_{\mathrm{SF}}$ to the values $n_{\uparrow} \simeq 1.01 n_{\mathrm{SF}}$ and $n_{\downarrow}=x_{c} n_{\uparrow} \simeq$ $0.44 n_{\mathrm{SF}}$, respectively, as one enters the normal phase. The discontinuity is an artifact of LDA applied to first order phase transitions. In Fig. 4 we plot $n_{\uparrow}$ and $n_{\downarrow}$ as well as the difference $n_{\uparrow}-n_{\downarrow}$ as a function of position in a spherical trap when $P=P_{c}$. These results, based on LDA, apply also to anisotropic traps through a simple

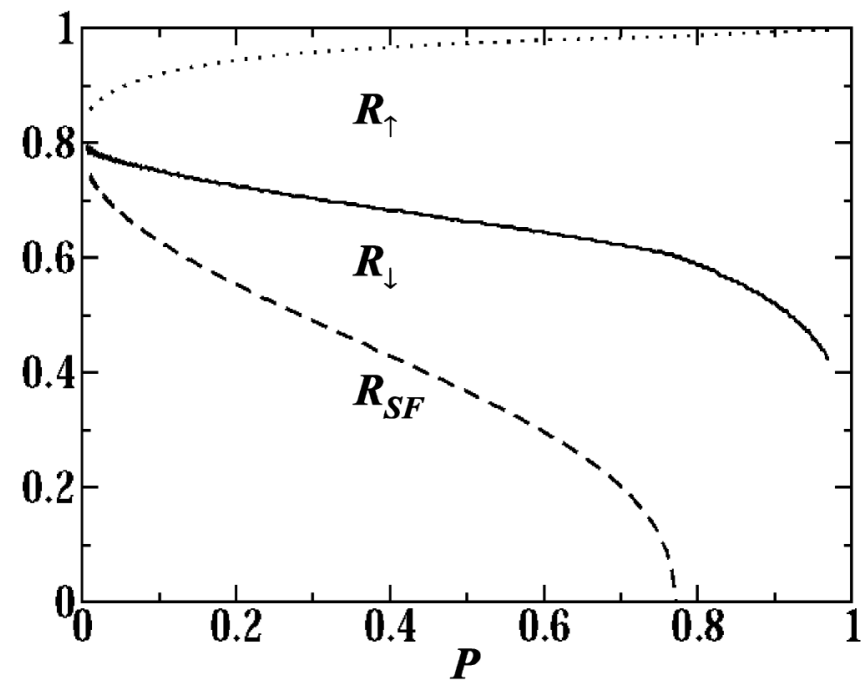

FIG. 3. Radii of the three phases in the trap in units of the radius $R_{\uparrow}^{0}=a_{\mathrm{ho}}\left(48 N_{\uparrow}\right)^{1 / 6}$ of a noninteracting fully polarized gas, where $a_{\text {ho }}$ is the harmonic oscillator length. 


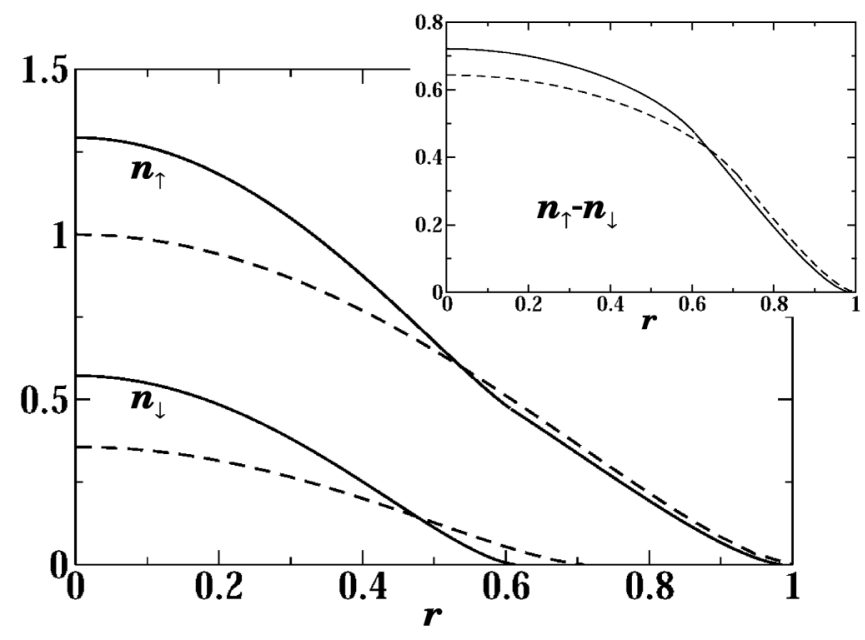

FIG. 4. Density profiles (solid lines) for the two spin components in a spherical harmonic trap at the critical total polarization $P_{c}=0.77$. The density profiles (dashed lines) for a noninteracting Fermi gas with the same polarization are also shown. The inset shows the density difference profile. Densities are given in units of the central density of the noninteracting gas. The radial coordinate is given in units of $R_{\uparrow}^{0}$ (see Fig. 3).

scaling transformation. We find that both the total density $n_{\uparrow}+n_{\downarrow}$ and the density difference $n_{\uparrow}-n_{\downarrow}$ increase monotonically towards the center [18]. If $P<P_{c}$, then $n_{\uparrow}=n_{\downarrow}$ in the central superfluid region.

In conclusion, we study the polarized Fermi gas at unitarity as a normal Fermi liquid composed of weakly interacting quasiparticles associated with the minority atoms. These have a quadratic dispersion and, in the $x \rightarrow$ 0 limit, have an effective mass $m^{*} \simeq 1.04(3) m$ with a binding energy $-3 / 5 E_{F \uparrow} \times 0.97(2)$, calculated with a FN-DMC approach. We derive an energy functional Eq. (2) with those parameters, assuming noninteracting quasiparticles, and using FN-DMC calculations at higher values of $x$, show that corrections to the energy functional are small, even for relatively high concentrations. Assuming that no polarized superfluid phases exist, we predict a normal-superfluid first order phase transition at a critical value $x_{c}=0.44$, corresponding, in the presence of harmonic trapping, to a critical total polarization $P_{c}=$ 0.77 . We also predict for small concentrations an increase by a factor of 1.23 of the frequency of the spin dipole mode with respect to the noninteracting value. A further application of the equation of state of the mixed phase could be, for example, the calculation of the dipole polarizability of the trapped Fermi gas [19].

We acknowledge useful discussions with A. Bulgac, I. Carusotto, A. J. Leggett, L.P. Pitaevskii, and N. Prokof'ev. We also acknowledge support by the Ministero dell'Istruzione, dell'Università e della Ricerca (MIUR).
[1] G. B. Partridge, W. Li, R. I. Kamar, Y. Liao, and R. G. Hulet, Science 311, 503 (2006); M. W. Zwierlein, C. H. Schunck, A. Schirotzek, and W. Ketterle, Nature (London) 442, 54 (2006).

[2] Y. Shin, M. W. Zwierlein, C. H. Schunck, A. Schirotzek, and W. Ketterle, Phys. Rev. Lett. 97, 030401 (2006).

[3] J. Carlson and S. Reddy, Phys. Rev. Lett. 95, 060401 (2005); P. Pieri and G. C. Strinati, Phys. Rev. Lett. 96, 150404 (2006); D. E. Sheehy and L. Radzihovsky, ibid. 96, 060401 (2006); J. Kinnunen, L. M. Jensen, and P. Torma, ibid. 96, 110403 (2006); M. Haque and H. T. C. Stoof, Phys. Rev. A 74, 011602 (2006); W. Yi and L.-M. Duan, Phys. Rev. A 73, 031604(R) (2006); 74, 013610 (2006); K. Machida, T. Mizushime, and M. Ichioka, condmat/0604339; M. M. Parish, F. M. Marchetti, A. Lamacraft, and B. D. Simons, cond-mat/0605744; T. N. De Silva and E. J. Mueller, Phys. Rev. Lett. 97, 120407 (2006); 97, 070402 (2006); F. Chevy, Phys. Rev. Lett. 96, 130401 (2006); M. Iskin and C. A. R. Sa de Melo, Phys. Rev. Lett. 97, 100404 (2006); C. Chien, Q. Chen, Y. He, and K. Levin, cond-mat/0605039; K. B. Gubbels, M. W. J. Romans, and H. T. C. Stoof, cond-mat/0606330.

[4] C.-H. Pao and S.-K. Yip, J. Phys. C 18, 5567 (2006).

[5] T.N. De Silva and E.J. Mueller, Phys. Rev. A 73, 051602(R) (2006).

[6] F. Chevy, cond-mat/0605751.

[7] A. Bulgac and M. M. Forbes, cond-mat/0606043.

[8] See, e.g., J. Bardeen, G. Baym, and D. Pines, Phys. Rev. 156, 207 (1967).

[9] Adding a $\downarrow$ atom to a gas of $\uparrow$ atoms in the Bose-Einstein condensation limit would lead instead to the formation of a molecule. We would then have a mixture of $\uparrow$ atoms and a Bose-Einstein condensate of molecules.

[10] G.E. Astrakharchik, J. Boronat, J. Casulleras, and S. Giorgini, Phys. Rev. Lett. 93, 200404 (2004).

[11] G.E. Astrakharchik, J. Boronat, J. Casulleras, and S. Giorgini, Phys. Rev. Lett. 95, 230405 (2005).

[12] F. AriasdeSaavedra, J. Boronat, A. Polls, and A. Fabrocini, Phys. Rev. B 50, 4248 (1994).

[13] J. Carlson, S. Y. Chang, V. R. Pandharipande, and K. E. Schmidt, Phys. Rev. Lett. 91, 050401 (2003).

[14] We do not take into account the possibility of a polarized superfluid which would modify the possible transitions.

[15] Upper and lower bounds for $\mu_{\downarrow} / \mu_{\uparrow}$ at the fully polarizedmixed and mixed-superfluid transitions (which are compatible with our values) were recently given in Refs. [6,7].

[16] See [5] for a discussion of the validity of LDA.

[17] One concern is the possibility that this mode may be overdamped due to the nonsuperfluid nature of the system. However, at $k_{B} T \ll E_{F \uparrow}$ we expect that the damping of the oscillation will be sufficiently small.

[18] A preliminary comparison with the profiles of [2] reveals a qualitative discrepancy: it is seen experimentally that the density difference has a minimum in the center also at high polarizations. This might be a signature of a polarized superfluid phase.

[19] A. Recati, I. Carusotto, C. Lobo, and S. Stringari, condmat/0605754. 